\title{
MORPHOLOGICAL AND GRAIN YIELD RESPONSES OF SOYBEAN (GLYCINE MAX L.) TO TIME AND INTENSITY OF DEFOLIATION UNDER APPLICATION OF AMINO ACID
}

\author{
NAJAFIKHAN-BEHBIN, H. ${ }^{1}-$ DADASHI, M. R. $.^{1 *}-$ FARAJI, A. $^{2}-$ SOLTANI, A. ${ }^{3}$ \\ ${ }^{1}$ Department of Agronomy, Gorgan Branch, Islamic Azad University, Gorgan, Iran \\ ${ }^{2}$ Horticulture and Agronomy Department, Golestan Agricultural and Natural Resources \\ Research and Education Center, Agricultural Research, Education and Extension Organization \\ (AREEO), Gorgan, Iran \\ ${ }^{3}$ Department of Plant Physiology, Faculty of Agriculture, University of Gorgan, Gorgan, Iran \\ *Corresponding author \\ e-mail:mdadashi730@yahoo.com \\ (Received $1^{\text {st }}$ Nov 2018; accepted $25^{\text {th }}$ Jan 2019)
}

\begin{abstract}
The use of amino acids (AA) to increase the uptake of nutrients has been increasing dramatically in recent years. This investigation was conducted in the two successive seasons of 2016 and 2017 on soybean in Golestan Province, Iran, to study the effect of time and intensity of defoliation on quantitative and qualitative characteristics of soybean (Glycine max L.) under the application of AA. A two-year factorial experiment was conducted based on randomized complete block design with three replications. The first factor included the defoliation intensity at three levels 0,50 and $100 \%$, the second factor included the defoliation time in five levels V1, V3, V5, V7, and R1 (growth stages), and the third factor was the application and non-application of AA. Results indicated that the effects of the defoliation time and intensity, as well as the foliar application of AA, were significant on morphological traits, yield and yield components, and oil and protein contents. In the quadruple interaction, the highest of grain yield (4040 kg.ha $\mathrm{h}^{-1}$ ) was observed in the application of AA under defoliation in V5 with 50\% intensity during the first year. The lowest value of this trait was achieved in non-application of AA under $100 \%$ defoliation in R1 growth stage during both years of the experiment (1585 and $1525 \mathrm{~kg}_{\text {.ha }}{ }^{-1}$, respectively). The maximum grain oil and protein yield were observed in $100 \%$ defoliation in V7 growth stage under AA application during the first-year experiment (978.5 and $1499 \mathrm{~kg}^{-h a}{ }^{-1}$, respectively). Also, the lowest value of the traits was observed in $100 \%$ defoliation in the V5 growth stage under non-application of AA during the second year (225.6 and $471.9 \mathrm{~kg} \cdot \mathrm{ha}^{-1}$, respectively). According to the present study, soybean is regarded generally as a defoliation-tolerant crop. Also, foliar application of AA could be used as an effective amendment for preventing defoliation injury in yield and yield components of soybean.
\end{abstract}

Keywords: foliar application, harvest index, oil content, pod, grain yield

\section{Introduction}

Soybean (Glycine $\max$ L.) is an important industrial legume as human and animal feed in the world with an average of 18-22\% oil, 38-56\% vegetable protein in its seeds. Soybean is the most widely grown oilseed in the world as a main or second crop (Arslan et al., 2018). Soybean is originated from China. Domestication and cultivation of soybean by humans in China date from about the $11^{\text {th }}$ century BC or a little earlier according to oldest records (Hymowitz and Shurtleff, 2005). The plant is introduced from the ancient world to the new world in the middle of the $17^{\text {th }}$ century and gained its worldwide importance at the beginning of the $20^{\text {th }}$ century (Arslan et al., 2018).

Plants are continuously exposed to biotic and abiotic stresses (Sadak et al., 2015). Plant response to defoliation depends on more than just the total amount of leaf area that 
is lost (Ahmadi et al., 2009). It is also known that defoliation intensity may vary along nutrient availability gradients and that defoliation may alter competition relationship between species (Alhamd and Alrababah, 2008). The ability of soybean to prevent substantial reduction in yield after the loss of leaves caused by defoliators and disease depends on several factors among including intensity of, the phenological stage of development at the time of defoliation, duration of defoliation, the ability of cultivar to tolerate or compensate for defoliation, and environmental conditions, especially rainfall, temperature and solar radiation (Nardino et al., 2016). Regarding the percentage of defoliation, research has established levels for the control of insect pests, when the defoliation is greater than $30 \%$ in the vegetative phase, or $15 \%$ in the reproductive phase. However, these recommendations are based on work done in the 70-80's decades. In this sense, another key issue for reduction of income is the degree to which defoliation reduces light interception by the canopy (Nardino et al., 2016). Earlier studies addressed the effect of leaf removal on growth and yield. In soybean, during the reproductive period, defoliation levels reduced the rate of the natural trend of losing leaf area. The yield was affected only by 67 and $100 \%$ defoliation applied at R6, while main agronomic traits such as date of harvesting maturity, plant loading, and height were not affected by defoliation (An et al., 2003). The defoliation of maize leaf up to 50\% at the time of feed shortage did not have adverse in grain and stover yield components of maize (Khaliliaqdam et al., 2012). During the reproductive period, levels of defoliation reduced the rate of the soybean natural trend of losing leaf area (Pickle and Caviness, 2006).

The amino acid (AA) is a well-known biostimulant which has positive effects on plant growth, yield and significantly mitigates the injuries caused by biotic or abiotic stresses (Kowalczyk and Zielony, 2008). Saeed et al. (2005) on soybean found that treatments of AA significantly improved growth parameters of shoots and fresh weight as well as pod yield. Foliar application of nutrients has been recognized by many researchers, as a very efficient method of plant nutrition (Stiegler et al., 2013). Amino acid foliar applications are biostimulants in plants because they enhance the nutrient uptake efficiency because of increases in the leaf cuticle permeability (Moreira and Moraes, 2017), better plant growth, and higher plant biomass and grain yield, and they reduce abiotic stresses (Mendes et al., 2016). Researches are still necessary to verify the effects from amino acid foliar fertilizers under intensity and time of defoliation to improved quality and quantity of soybean plant. The objective of this study was to evaluate the influence of intensity and time of defoliation under amino acid application on yield and yield components and grain oil and protein contents of the soybean plant.

\section{Materials and methods}

\section{Experimental design, site, and soil chemical analysis}

In order to evaluate the effect of intensity and time of defoliation on quantitative and qualitative traits of soybean (Katol cultivar) under application of amino acid condition, a two-year factorial experiment was used based on randomized complete block design with three replications in, Golestan Province, Iran (Aliabad Katoul city; lat. 34 54', long. 54 $56^{\prime} \mathrm{E}$, altitude $142 \mathrm{~m}$ ) during two consecutive years 2015-16 and 2016-17. The average annual minimum and maximum temperature and rainfall were 12.5 and $23.6^{\circ} \mathrm{C}$, and $182 \mathrm{~mm}$ (Table 1). The first factor included the intensity of defoliation soybean leaf in three levels 0,50 and $100 \%$, the second factor included time of 
defoliation in five levels (V1, V3, V5, V7, and R1; soybean growth stages), and the third factor was the use and non-use of amino acids.

Table 1. Monthly weather characteristics of the test area in the year of implementation

\begin{tabular}{c|c|c|c|c|c|c|c|c|c|c|c|c}
\hline Parameters & Apr & May & Jun & Mar & Aug & Sep & Oct & Nov & Dec & Jan & Feb & Mar \\
\hline Maximum air temperature $\left({ }^{\circ} \mathrm{C}\right)$ & 20.1 & 27.6 & 31.4 & 34.7 & 35.0 & 32.7 & 25.0 & 18.8 & 13.6 & 13.0 & 9.9 & 16.8 \\
Minimum air temperature $\left({ }^{\circ} \mathrm{C}\right)$ & 9.6 & 15.2 & 18.9 & 22.2 & 22.2 & 20.3 & 13.8 & 9.1 & 2.6 & 3.4 & 2.8 & 5.2 \\
Air temperature mean $\left({ }^{\circ} \mathrm{C}\right)$ & 14.9 & 21.4 & 25.2 & 28.5 & 28.6 & 26.5 & 19.4 & 13.9 & 8.1 & 8.2 & 6.4 & 11.0 \\
Relative humidity $(\%)$ & 77 & 74 & 68 & 64 & 62 & 64 & 71 & 77 & 73 & 72 & 78 & 74 \\
Rainfall (mm) & 147.4 & 46.6 & 30.5 & 22.2 & 31.3 & 68.9 & 36.2 & 73.0 & 53.6 & 6.7 & 100.3 & 44.1 \\
Total number of rainy days & 16 & 14 & 7 & 8 & 6 & 8 & 7 & 9 & 13 & 5 & 18 & 9 \\
Total amount of evaporation (mm) & 72.4 & 145.3 & 191.4 & 198.8 & 231.0 & 183.7 & 109.8 & 64.6 & 53.4 & 41.3 & 29.9 & 64.0 \\
The number of frosty days & 0 & 0 & 0 & 0 & 0 & 0 & 0 & 0 & 9 & 0 & 3 & 2 \\
\hline
\end{tabular}

Agronomical operations were carried out in accordance with the tradition of the area. The previous planting on the farm was wheat. In order to determine some physical and chemical properties of the soil, a sample from $0-30 \mathrm{~cm}$ depth was prepared and sent to the laboratory and the amount of fertilizer was taken according to the laboratory's recommendations (Table 2). After fertilization, cultivation was carried out with a soybean row known as Fatahi's row planter (is a famous brand). The size of each plot was $2 \times 3 \mathrm{~m}$ (with five rows). The distance between blocks and the distance plot in each block were 2 and $1 \mathrm{~m}$, respectively. The pesticide used in the first stage consisted of Thiodicarb (1 kg.ha-1) and secondly, 2 L/ha of Chlorpyrifos and thus, $250 \mathrm{cc}$ Imidacloprid. Hand weeding weeds and 5 irrigations steps were performed.

Table 2. Physicochemical properties of soil in the experimental farm at depths of 0 to $30 \mathrm{~cm}$

\begin{tabular}{c|c|c|c|c|c|c|c|c|c|c}
\hline $\begin{array}{c}\text { Soil } \\
\text { texture }\end{array}$ & $\begin{array}{c}\text { Sand } \\
(\boldsymbol{\%})\end{array}$ & $\begin{array}{c}\text { Silt } \\
(\boldsymbol{\%})\end{array}$ & $\begin{array}{c}\text { Clay } \\
(\boldsymbol{\%})\end{array}$ & $\begin{array}{c}\mathbf{K} \\
(\mathbf{m g} / \mathbf{k g})\end{array}$ & $\begin{array}{c}\mathbf{P} \\
(\mathbf{m g} / \mathbf{k g})\end{array}$ & $\begin{array}{c}\mathbf{N} \text { total } \\
(\boldsymbol{\%})\end{array}$ & $\begin{array}{c}\text { Organic } \\
\text { carbon }(\boldsymbol{\%})\end{array}$ & $\begin{array}{c}\text { Percentage of } \\
\text { neutralized matter }\end{array}$ & $\mathbf{p H}$ & $\begin{array}{c}\mathbf{E C} \\
\left(\mathbf{d S} / \mathbf{m}^{2}\right)\end{array}$ \\
\hline $\begin{array}{c}\text { Clay } \\
\text { loam }\end{array}$ & 22 & 50 & 28 & 261 & 8.8 & 0.15 & 1.46 & 12.5 & 7.4 & 2.4 \\
\hline
\end{tabular}

Underuse of AA conditions, spraying with the recommended dose of $1 \mathrm{~kg}$ per 1000 liters of water (250 g.ha ${ }^{-1}$ ) was performed at V3, V5, V7, and R1 growth stages (Fig. 1). The AA used by the Nutramin-WSP brand is Biomega, which contains 14-15\% nitrogen, $0.3-0.7 \%$ calcium, $0.4-1.2 \%$ phosphate, $1.1-1.5 \%$ potassium, $0.3-0.6 \%$ ammonium, and $90 \%$ amino acids. The intensity and time of defoliation (by hand) was done also determined by dividing the soybean morphology stages. For this purpose, at $0 \%$ level (no defoliation of leaves), 50\% (defoliation of 50\% the leaves) and $100 \%$ (defoliation of $100 \%$ the leaves) was performed (Fig. 2).

\section{Yield and yield components}

To measure morphological and yield characteristics, at the R7 stage, five samplings from each experimental plot were examined for yield components (with marginal effect) and plant height: the height of the first pod from the surface, number of branches, number of pods, number of seeds per pod, and 100-seed weight were measured. In order to determine the grain yield after removing the marginal effects, two rows of middle 
were harvested for grain yield. In other words, yield components were measured according to plant levels, but grain yield was measured according to surface levels. After calculating the biological yield, the harvest index (from grain yield to biological yield) was also determined (Divsalar et al., 2015).

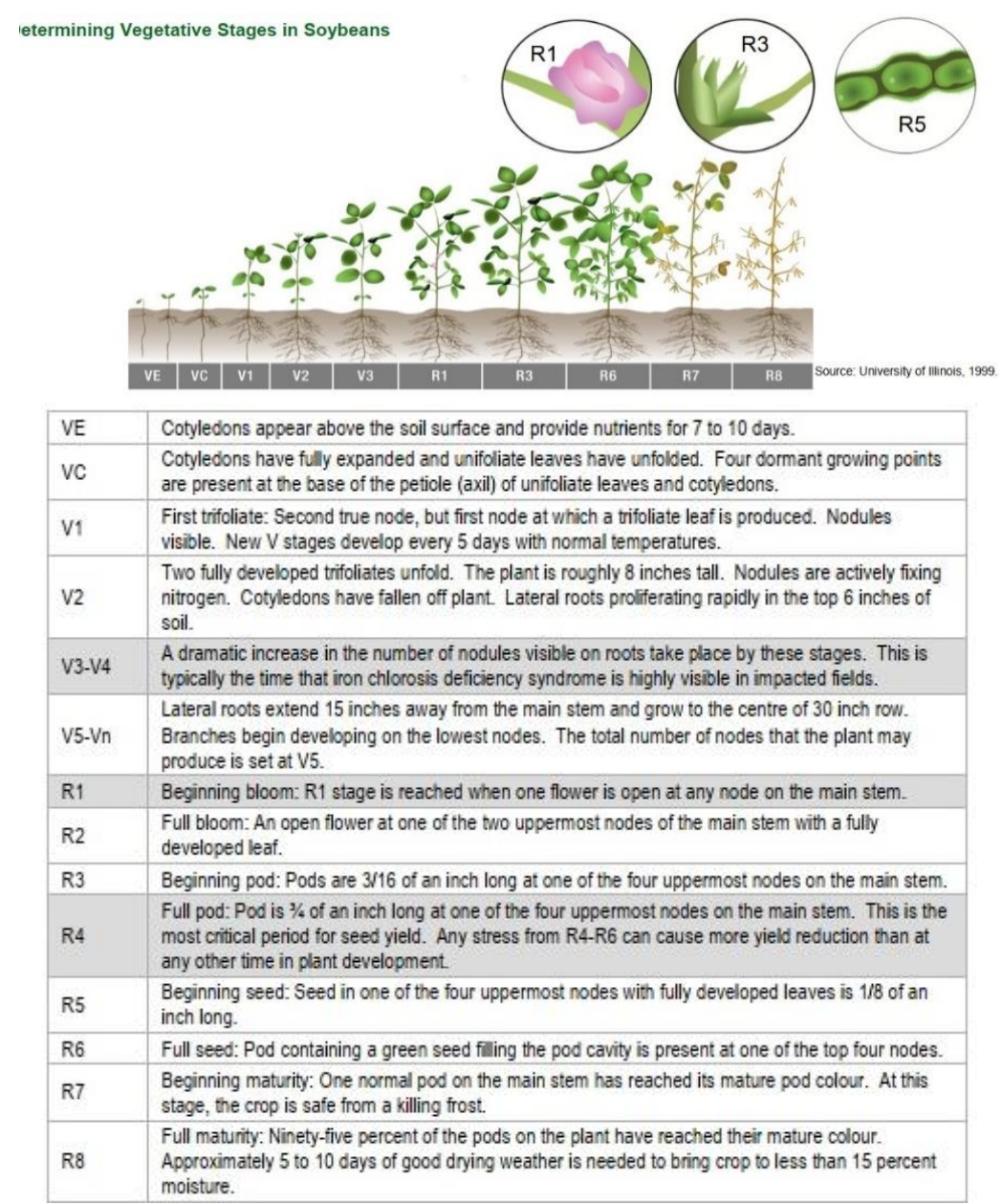

Figure 1. Determining vegetative stage in soybeans

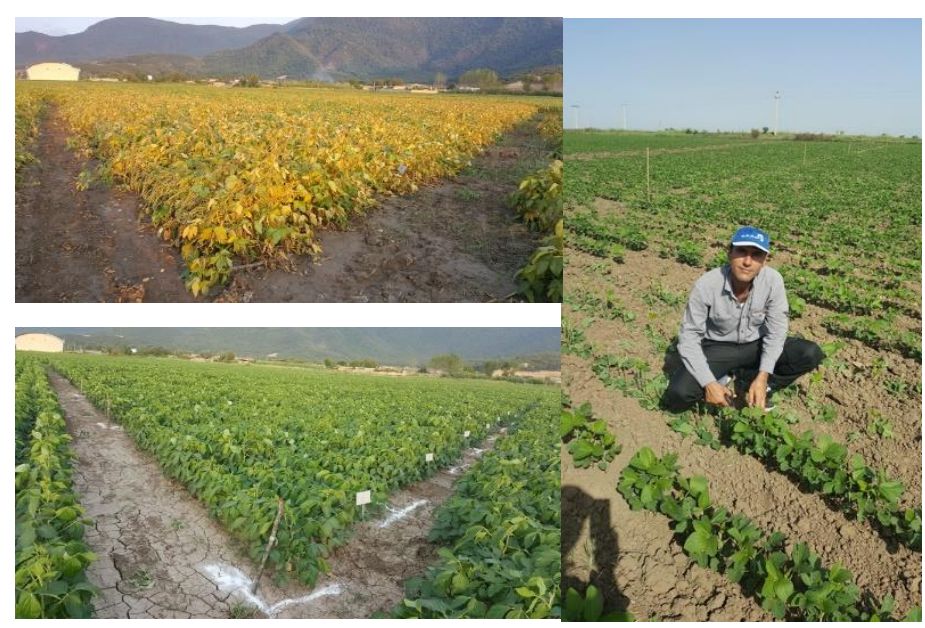

Figure 2. Experimental site and planting farm 


\section{Grain oil and protein content}

To measure the oil content and seed protein, about 50 seeds were randomly selected from each treatment. After powdered, the percentage of oil by Soxhlet method and protein percentage were also measured by Kjeldahl method (Divsalar et al., 2015). To calculate oil yield and protein yield, oil percentage and protein percentage were multiplied in grain yield, respectively.

\section{Statistical analysis}

Analysis of variance was performed to test for statistical differences among the treatments, and means were statistically analyzed using least significant difference test (LSD) at $\mathrm{P}<0.05$ level of significance by using SAS software (Statistical Analysis Software, 9.2).

\section{Results}

\section{Plant height}

Results indicated that the effect of year (Y), amino acid (AA), defoliation time (DT), and defoliation intensity (DI) were significant on plant height (Table 3). The maximum plant height was achieved in first Y $(93.02 \mathrm{~cm})$, foliar application of AA $(92.16 \mathrm{~cm})$, DT in V1 $(94.03 \mathrm{~cm}$ ), and DI in 0 and 50\% (96.0 and $92.8 \mathrm{~cm}$, respectively). Mean comparison results showed the interaction effects of $\mathrm{Y} \times \mathrm{AA}$ and $\mathrm{DT} \times \mathrm{DI}$ were significant on plant height. The highest and lowest plant height were observed DT in V1 and DT in control treatment $(98.16 \mathrm{~cm})$ and DT in V5, V7, and R1 under DI in $100 \%$ (74.14, 72.33, and $71.66 \mathrm{~cm}$, respectively) (Fig. 3A). In interaction $\mathrm{Y} \times \mathrm{AA}$, the maximum this trait was observed in the first $Y$ under application of AA $(99.8 \mathrm{~cm})$, and the lowest value of this trait was achieved in the second $Y$ under non-application of AA $(84.4 \mathrm{~cm})($ Fig. 3B).
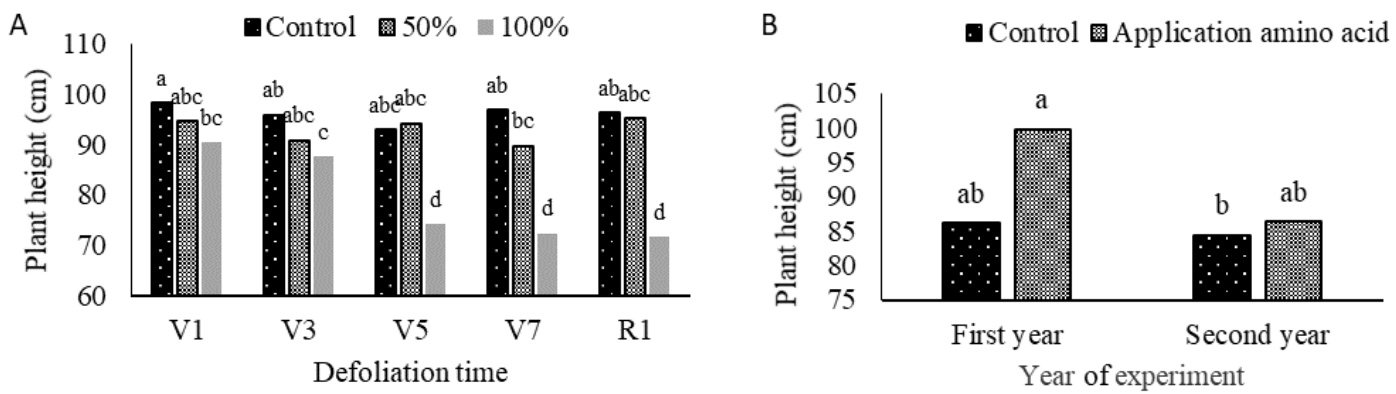

Figure 3. Effect of intensity (0, 50, and 100\%) and time (V1, V3, V5, V7, and R1) of defoliation (A); and defoliation time (VI,V3,V5, V7, and R1) and amino acid (control and application) (B) on plant height of soybean (columns with different letters are significantly different at $P=0.05$, using LSD test)

\section{First pod height from the surface}

In the current study, the effects of Y, DT, and interaction DT $\times$ DI were significant on the first pod height from the surface. The highest means of this trait was observed in the first $\mathrm{Y}(21.18 \mathrm{~cm})$ and DT in R1 $(22.0 \mathrm{~cm})$ (Table 3). Mean comparison of 
interaction DT $\times$ DI showed the highest value of the trait was achieved in DT in R1 and DI in $100 \%$ (24.75 cm) (Fig. 4).

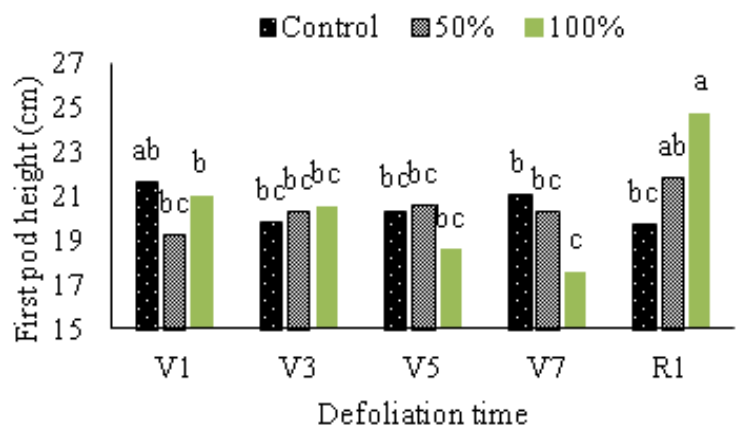

Figure 4. Effect of intensity (0, 50, and 100\%) and time (V1, V3, V5, V7, and R1) of defoliation on first pods height from the surface (columns with different letters are significantly different at $P=0.05$, using LSD test)

\section{Number of branches}

The effects of $\mathrm{Y}, \mathrm{AA}, \mathrm{DT}, \mathrm{Y} \times \mathrm{A}$, and $\mathrm{DT} \times \mathrm{DI}$ was significant on number of lateral branches. Also, the effects of $\mathrm{Y}, \mathrm{DT}, \mathrm{DI}, \mathrm{Y} \times \mathrm{DI}$, and $\mathrm{AA} \times \mathrm{DI}$ were significant on number of main branches (Table 3). The highest number of lateral branch was observed DT in V3 under DI in $100 \%$ (6.93 no.). The lowest value of this trait was achieved in DT in R1 under DI in $100 \%$ (3.32 no.) (Fig. 5A). In the interaction DT $\times$ AA, the highest number of lateral branch was observed first $Y$ under non-application of AA (6.98 no.) and the lowest value of this trait was observed in the second $\mathrm{Y}$ under non-application AA (4.15 no.) (Fig. 5B).
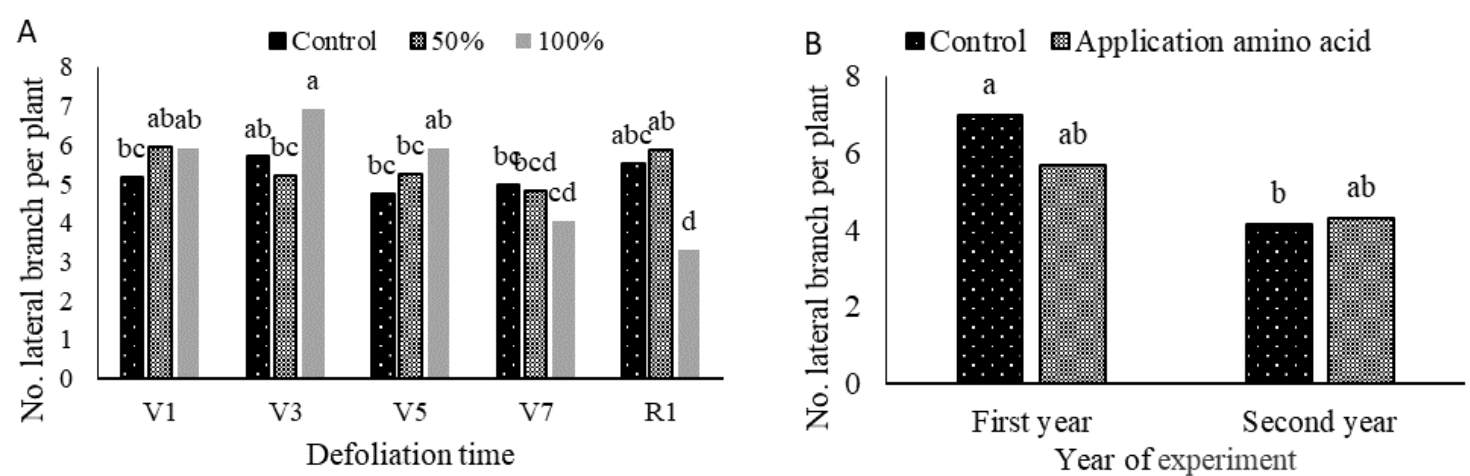

Figure 5. Effect of intensity (0, 50, and 100\%) and time (V1, V3, V5, V7, and R1) of defoliation (A); and defoliation time (VI,V3,V5, V7, and R1) and amino acid (control and application) (B) on number of lateral branch per plant (columns with different letters are significantly different at $P=0.05$, using LSD test)

\section{Number of the total pods}

Results showed that the effect of Y, DT, and DI was significant on number of total pods (Table 3). The highest of the number of total pods was observed in the first $\mathrm{Y}$ 
(69.05 no.), DT in V1, V3, and V5 $(64.8,67.0$, and 62.3 no., respectively), and DI in $50 \%$ (63.4 no.) (Table 3).

\section{Number of pod free grain}

The effects of $\mathrm{Y}$ and $\mathrm{Y} \times \mathrm{AA}$ was significant on number of pod free grain (Table 4). The highest number of pod free grain was observed in first $\mathrm{Y}$ (5.83 no.). In interaction $\mathrm{Y} \times \mathrm{AA}$, the highest number of pods free grain was observed in the first $\mathrm{Y}$ under nonapplication and application of amino acid (4.97 and 6.69 no., respectively). The lowest value of the trait was achieved in the second $\mathrm{Y}$ under both amino acid treatments (Fig. 6).

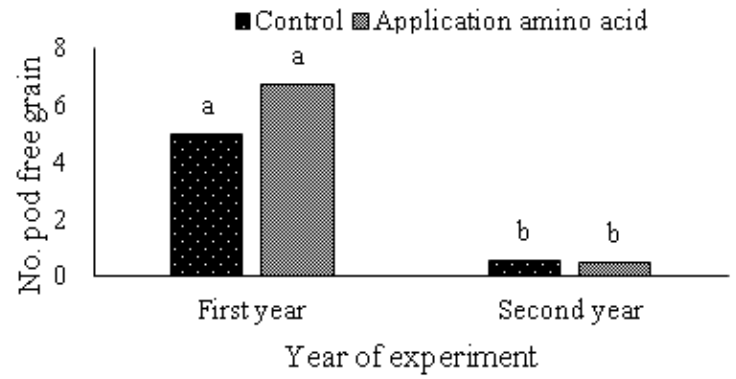

Figure 6. Effect of the year (first and second) and amino acid (control and application) on number of pod free grain of soybean (columns with different letters are significantly different at $P=0.05$, using LSD test)

\section{Grain number per plant}

Results indicated that the effects of Y, DT, DI, and DT $\times$ DI was significant on grain number of soybean (Table 4). The first $\mathrm{Y}$ had superiority in term of grain number in compared to the second Y. Among the DT treatments, defoliation in the V1 growth stage had the highest grain number (152.2 no. per plant). By increasing defoliation intensity observed reduction the grain number per plant, so that the zero and $100 \%$ intensity of defoliation has the highest and lowest means of the trait (147.7 and 122.5 no per plant, respectively). In the interaction $\mathrm{DT} \times \mathrm{DI}$, the highest grain number was achieved in V1 and V5 growth stages under 50\% defoliation (164.7 and 163.5 no., respectively). The lowest value of the trait was observed in $\mathrm{R} 1$ growth stages under $100 \%$ defoliation (74.7 no.) (Fig. 7).

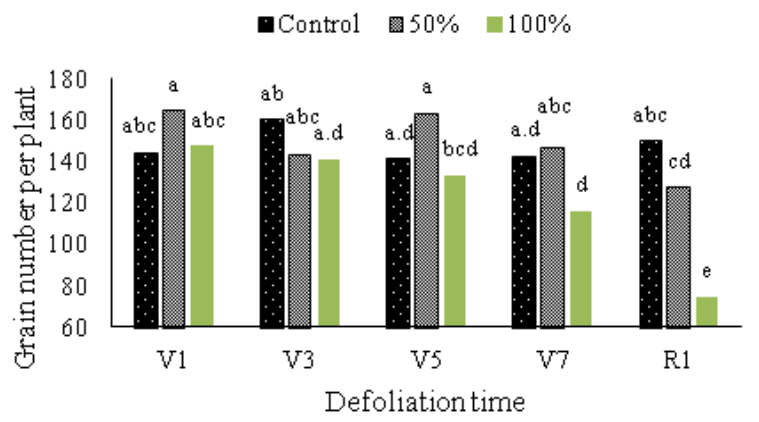

Figure 7. Effect of intensity (0, 50, and 100\%) and time (V1, V3, V5, V7, and R1) of defoliation on grain number of soybean (columns with different letters are significantly different at

$$
P=0.05 \text {, using LSD test) }
$$


Table 3. Analysis of variance indicating the effects of intensity and time of defoliation on plant height, first pod height, number of lateral branches, number of the main branch, and number total pods of soybean under application of amino acid

\begin{tabular}{|c|c|c|c|c|c|}
\hline Treatments & $\begin{array}{l}\text { Plant height } \\
\text { (cm) }\end{array}$ & $\begin{array}{l}\text { First pod } \\
\text { height }(\mathrm{cm})\end{array}$ & $\begin{array}{c}\text { No. lateral } \\
\text { branch per } \\
\text { plant }\end{array}$ & $\begin{array}{c}\text { No. main } \\
\text { branch per } \\
\text { plant }\end{array}$ & $\begin{array}{c}\text { No. total pod } \\
\text { per plant }\end{array}$ \\
\hline \multicolumn{6}{|l|}{ Year $(Y)$} \\
\hline First & $93.02 \mathrm{a}$ & $21.18 \mathrm{a}$ & $6.33 \mathrm{a}$ & $33.85 \mathrm{a}$ & $69.05 \mathrm{a}$ \\
\hline Second & $85.75 \mathrm{~b}$ & $19.73 \mathrm{~b}$ & $4.25 \mathrm{~b}$ & $30.40 \mathrm{~b}$ & $52.31 \mathrm{~b}$ \\
\hline $\operatorname{LSD}(P=0.05)$ & 2.75 & 1.18 & 0.57 & 1.69 & 5.75 \\
\hline \multicolumn{6}{|l|}{ Amino acid (AA) } \\
\hline Control & $85.50 \mathrm{~b}$ & $20.98 \mathrm{a}$ & $5.85 \mathrm{a}$ & $31.30 \mathrm{a}$ & $57.90 \mathrm{a}$ \\
\hline Application & $92.16 \mathrm{a}$ & $20.08 \mathrm{a}$ & $4.89 \mathrm{~b}$ & $32.71 \mathrm{a}$ & $62.67 \mathrm{a}$ \\
\hline $\operatorname{LSD}(P=0.05)$ & 2.79 & 1.20 & 0.58 & 1.72 & 5.83 \\
\hline \multicolumn{6}{|l|}{ Defoliation time (DT) } \\
\hline $\mathrm{V} 1$ & $94.3 \mathrm{a}$ & $20.6 \mathrm{ab}$ & $5.68 \mathrm{ab}$ & $34.7 \mathrm{a}$ & $64.8 \mathrm{a}$ \\
\hline V3 & $91.4 \mathrm{ab}$ & $20.1 \mathrm{~b}$ & $5.95 \mathrm{a}$ & $32.1 \mathrm{abc}$ & $67.0 \mathrm{a}$ \\
\hline V5 & $87.1 \mathrm{bc}$ & $19.4 \mathrm{~b}$ & $5.31 \mathrm{abc}$ & $32.8 \mathrm{ab}$ & $62.3 \mathrm{a}$ \\
\hline V7 & $86.0 \mathrm{c}$ & $19.9 \mathrm{~b}$ & $4.62 \mathrm{c}$ & $30.8 \mathrm{bc}$ & $58.0 \mathrm{ab}$ \\
\hline $\mathrm{R} 1$ & $87.9 \mathrm{bc}$ & $22.0 \mathrm{a}$ & $4.90 \mathrm{bc}$ & $30.0 \mathrm{c}$ & $51.0 \mathrm{~b}$ \\
\hline $\operatorname{LSD}(P=0.05)$ & 4.36 & 1.87 & 0.90 & 2.68 & 9.09 \\
\hline \multicolumn{6}{|l|}{ Defoliation intensity (DI) (\%) } \\
\hline 0 & $96.0 \mathrm{a}$ & $20.4 \mathrm{a}$ & $5.23 \mathrm{a}$ & $34.6 \mathrm{a}$ & $62.5 \mathrm{ab}$ \\
\hline 50 & $92.8 \mathrm{a}$ & $20.4 \mathrm{a}$ & $5.42 \mathrm{a}$ & $34.8 \mathrm{a}$ & $63.4 \mathrm{a}$ \\
\hline 100 & $79.2 \mathrm{~b}$ & $20.4 \mathrm{a}$ & $5.23 \mathrm{a}$ & $26.9 \mathrm{~b}$ & $56.0 \mathrm{~b}$ \\
\hline $\operatorname{LSD}(P=0.05)$ & 3.37 & 1.45 & 0.70 & 2.08 & 7.04 \\
\hline \multicolumn{6}{|l|}{ Interaction effect } \\
\hline $\mathrm{Y} \times \mathrm{A}$ & $* *$ & NS & $* *$ & NS & NS \\
\hline $\mathrm{Y} \times \mathrm{DT}$ & $*$ & NS & NS & NS & NS \\
\hline $\mathrm{Y} \times \mathrm{DI}$ & NS & NS & NS & $*$ & NS \\
\hline $\mathrm{AA} \times \mathrm{DT}$ & NS & NS & NS & NS & NS \\
\hline $\mathrm{AA} \times \mathrm{DI}$ & NS & NS & NS & $*$ & NS \\
\hline $\mathrm{DT} \times \mathrm{DI}$ & $* *$ & $*$ & $* *$ & NS & NS \\
\hline $\mathrm{Y} \times \mathrm{AA} \times \mathrm{DT}$ & NS & NS & NS & NS & NS \\
\hline $\mathrm{Y} \times \mathrm{AA} \times \mathrm{DI}$ & NS & NS & NS & NS & NS \\
\hline $\mathrm{Y} \times \mathrm{DT} \times \mathrm{DI}$ & NS & NS & NS & NS & NS \\
\hline $\mathrm{AA} \times \mathrm{DT} \times \mathrm{DI}$ & NS & NS & NS & NS & NS \\
\hline $\mathrm{Y} \times \mathrm{AA} \times \mathrm{DT} \times \mathrm{DI}$ & NS & NS & NS & NS & NS \\
\hline
\end{tabular}

LSD: least significant difference; NS: non-significant. Columns with different letters are significantly different at $\mathrm{P}=0.05$, using LSD test

\section{0-grain weight}

The effects of $\mathrm{Y}, \mathrm{AA}, \mathrm{DT}, \mathrm{DI}, \mathrm{Y} \times \mathrm{AA}$, and $\mathrm{Y} \times \mathrm{AA} \times \mathrm{DT} \times \mathrm{DI}$ was significant on 100 grain weight (Table 4). In the first year (19.98 g), non-application of AA (20.01 g), defoliation in V1 growth stage (20 g), and control treatment of defoliation intensity (20.11 g) showed the highest mean of 100-grain weight (Table 4). In the quadruple 
interaction, the highest 100-grain weight was observed in control DI in V3 growth stage under non-application of AA during the first year $(20.8 \mathrm{~g})$ and lowest was achieved in $100 \%$ defoliation of leaf in V5 growth stage under application of AA during the second year (18.58 g) (Table 5).

Table 4. Analysis of variance indicating the effects of intensity and time of defoliation on no. pods free seed per plant, no. grain per plant, 100-grain weight, biological yield, grain yield, and harvest index of soybean under application of amino acid

\begin{tabular}{|c|c|c|c|c|c|c|}
\hline Treatments & $\begin{array}{l}\text { No. pods } \\
\text { free grain } \\
\text { per plant }\end{array}$ & $\begin{array}{l}\text { No. grain } \\
\text { per plant }\end{array}$ & $\begin{array}{l}\text { 100-grain } \\
\text { weight }(g)\end{array}$ & $\begin{array}{c}\text { Biological } \\
\text { yield } \\
\left(\mathrm{kg}^{\prime} \mathrm{ha}^{-1}\right)\end{array}$ & $\begin{array}{c}\text { Grain } \\
\text { yield } \\
\left(\text { kg.ha }^{-1}\right)\end{array}$ & $\begin{array}{c}\text { Harvest } \\
\text { index }(\%)\end{array}$ \\
\hline \multicolumn{7}{|l|}{ Year $(Y)$} \\
\hline First & $5.83 \mathrm{a}$ & $150.2 \mathrm{a}$ & $19.98 \mathrm{a}$ & $9698.3 \mathrm{a}$ & $3233.8 \mathrm{a}$ & $33.40 \mathrm{~b}$ \\
\hline Second & $0.49 \mathrm{~b}$ & $129.4 \mathrm{~b}$ & $19.61 \mathrm{~b}$ & 8059.7 b & $2795.6 \mathrm{~b}$ & $34.56 \mathrm{a}$ \\
\hline $\operatorname{LSD}(P=0.05)$ & 0.54 & 9.87 & 0.15 & 356.4 & 106.6 & 0.99 \\
\hline \multicolumn{7}{|l|}{ Amino acid (AA) } \\
\hline Control & $3.19 \mathrm{a}$ & $134.9 \mathrm{a}$ & $20.01 \mathrm{a}$ & $8611.0 \mathrm{~b}$ & $2832.1 \mathrm{~b}$ & $32.93 \mathrm{~b}$ \\
\hline Application & $3.14 \mathrm{a}$ & $143.3 \mathrm{a}$ & $19.64 \mathrm{~b}$ & $9070.5 \mathrm{a}$ & $3145.1 \mathrm{a}$ & $34.73 \mathrm{a}$ \\
\hline $\operatorname{LSD}(P=0.05)$ & 0.54 & 10.01 & 0.15 & 361.5 & 108.1 & 1.00 \\
\hline \multicolumn{7}{|l|}{ Defoliation time (DT) } \\
\hline V1 & $3.50 \mathrm{a}$ & $152.2 \mathrm{a}$ & $20.0 \mathrm{a}$ & $9378.5 \mathrm{a}$ & $3290.4 \mathrm{a}$ & $35.4 \mathrm{a}$ \\
\hline V3 & $3.41 \mathrm{a}$ & $148.1 \mathrm{ab}$ & $19.8 \mathrm{ab}$ & $9204.9 \mathrm{a}$ & $3141.3 \mathrm{ab}$ & $34.2 \mathrm{ab}$ \\
\hline V5 & $3.02 \mathrm{a}$ & $146.0 \mathrm{ab}$ & $19.8 \mathrm{ab}$ & 8997.9 a & $2978.0 \mathrm{bc}$ & $33.0 \mathrm{~b}$ \\
\hline V7 & $2.88 \mathrm{a}$ & $135.2 \mathrm{~b}$ & $19.7 \mathrm{~b}$ & $8418.1 \mathrm{~b}$ & $2850.2 \mathrm{c}$ & $33.8 \mathrm{~b}$ \\
\hline $\mathrm{R} 1$ & $3.00 \mathrm{a}$ & $117.4 \mathrm{c}$ & $19.6 \mathrm{~b}$ & $8395.8 \mathrm{~b}$ & $2813.6 \mathrm{c}$ & $33.2 \mathrm{~b}$ \\
\hline $\operatorname{LSD}(P=0.05)$ & 0.85 & 15.6 & 0.24 & 563.5 & 168.6 & 1.57 \\
\hline \multicolumn{7}{|l|}{ Defoliation intensity (DI) (\%) } \\
\hline 0 & $3.00 \mathrm{a}$ & $147.7 \mathrm{a}$ & $20.11 \mathrm{a}$ & $9548.8 \mathrm{a}$ & 3299.8 a & $34.85 \mathrm{a}$ \\
\hline 50 & $3.03 \mathrm{a}$ & $149.1 \mathrm{a}$ & $19.84 \mathrm{~b}$ & $9300.0 \mathrm{a}$ & $3213.8 \mathrm{a}$ & $34.80 \mathrm{a}$ \\
\hline 100 & $3.46 \mathrm{a}$ & $122.5 \mathrm{~b}$ & $19.44 \mathrm{c}$ & $7788.3 \mathrm{~b}$ & $2530.5 \mathrm{~b}$ & $32.30 \mathrm{~b}$ \\
\hline $\operatorname{LSD}(P=0.05)$ & 0.66 & 12.09 & 0.18 & 436.5 & 130.6 & 1.21 \\
\hline \multicolumn{7}{|l|}{ Interaction effect } \\
\hline $\mathrm{Y} \times \mathrm{AA}$ & $* *$ & NS & $* *$ & NS & NS & NS \\
\hline $\mathrm{Y} \times \mathrm{DT}$ & NS & NS & NS & $* *$ & $* *$ & NS \\
\hline $\mathrm{Y} \times \mathrm{DI}$ & NS & NS & NS & NS & NS & $*$ \\
\hline $\mathrm{AA} \times \mathrm{DT}$ & NS & NS & NS & NS & NS & NS \\
\hline $\mathrm{AA} \times \mathrm{DI}$ & NS & NS & NS & NS & NS & NS \\
\hline $\mathrm{DT} \times \mathrm{DI}$ & NS & $* *$ & NS & $* *$ & $* *$ & $*$ \\
\hline $\mathrm{Y} \times \mathrm{AA} \times \mathrm{DT}$ & NS & NS & NS & NS & NS & NS \\
\hline $\mathrm{Y} \times \mathrm{AA} \times \mathrm{DI}$ & NS & NS & NS & NS & NS & NS \\
\hline $\mathrm{Y} \times \mathrm{DT} \times \mathrm{DI}$ & NS & NS & NS & $*$ & $*$ & NS \\
\hline $\mathrm{A} \times \mathrm{DT} \times \mathrm{DI}$ & NS & NS & NS & NS & NS & NS \\
\hline $\mathrm{Y} \times \mathrm{AA} \times \mathrm{DT} \times \mathrm{DI}$ & NS & NS & $* *$ & $*$ & $* *$ & NS \\
\hline
\end{tabular}

LSD: least significant difference; NS: non-significant. Columns with different letters are significantly different at $\mathrm{P}=0.05$, using LSD test 


\section{Biological yield}

Results indicated that the effects of $\mathrm{Y}, \mathrm{AA}, \mathrm{DT}, \mathrm{DI}, \mathrm{Y} \times \mathrm{DT}, \mathrm{DT} \times \mathrm{DI}, \mathrm{Y} \times \mathrm{DT} \times \mathrm{DI}$, and $\mathrm{Y} \times \mathrm{AA} \times \mathrm{DT} \times \mathrm{DI}$ were significant on biological yield (Table 4$)$. The highest mean of biological yield was observed in the first Y $\left(9698.3 \mathrm{~kg} \cdot \mathrm{ha}^{-1}\right)$, the application of AA $\left(9070.5 \mathrm{~kg} \cdot \mathrm{ha}^{-1}\right)$. Also, defoliation in V1, V3, and V5 growth stages under intensity 0 and 50\% showed the maximum biological yield (Table 4). In the quadruple interaction, the highest of biological yield was observed in the application of AA under defoliation in V1 with $50 \%$ intensity during the first year $\left(11750 \mathrm{~kg}^{-h^{-1}}\right)$ and also, in the application of AA under defoliation in V5 with 50\% intensity during the first year $\left(11733.3 \mathrm{~kg} \cdot \mathrm{ha}^{-1}\right)$. Results indicated that the lowest mean of the trait was achieved in noapplication of AA under $100 \%$ defoliation in the R1 growth stage during second $\mathrm{Y}$ $\left(5225 \mathrm{~kg} \cdot \mathrm{ha}^{-1}\right)($ Table 5).

Table 5. Interaction effects of year, amino acid, defoliation time, and defoliation intensity on 100-grain weight, biological yield, and grain yield of soybean

\begin{tabular}{|c|c|c|c|c|c|c|}
\hline Year & $\begin{array}{c}\text { Amino } \\
\text { acid }\end{array}$ & $\begin{array}{l}\text { Defoliation } \\
\text { time }\end{array}$ & $\begin{array}{c}\text { Defoliation } \\
\text { intensity }\end{array}$ & $\begin{array}{c}\text { 100-grain weight } \\
\text { (g) }\end{array}$ & $\begin{array}{l}\text { Biological yield } \\
\left(\mathrm{kg}^{\circ} \mathrm{ha}^{-1}\right)\end{array}$ & $\begin{array}{l}\text { Grain yield } \\
\left(\text { kg.ha }^{-1}\right)\end{array}$ \\
\hline \multirow{9}{*}{ 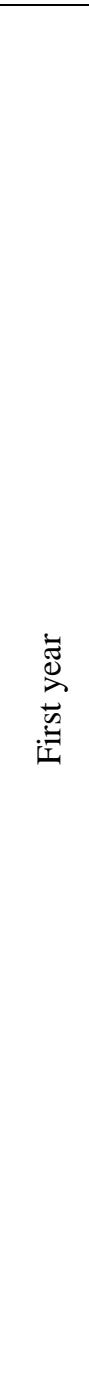 } & \multirow{5}{*}{$\begin{array}{l}\overrightarrow{0} \\
\dot{0} \\
\dot{0}\end{array}$} & V1 & $\begin{array}{c}\text { Control } \\
50 \% \\
100 \% \\
\end{array}$ & $\begin{array}{c}20.5 \pm 1.2 \text { a.d } \\
19.93 \pm 0.21 \text { c.m } \\
20.07 \pm 0.46 \text { b.k }\end{array}$ & $\begin{array}{c}9616.7 \pm 1200.3 \text { b.l } \\
10816.7 \pm 1350.3 \text { a.d } \\
9900 \pm 1525.6 \text { b.k }\end{array}$ & \begin{tabular}{|c|}
$3025 \pm 190.8$ f.o \\
$3615 \pm 258.2$ a.f \\
$3058.3 \pm 191.1$ f.o \\
\end{tabular} \\
\hline & & V3 & $\begin{array}{c}\text { Control } \\
50 \% \\
100 \% \\
\end{array}$ & $\begin{array}{c}\mathbf{2 0 . 8} \pm \mathbf{0 . 5 6} \mathbf{~ a} \\
20.4 \pm 0.75 \text { a.e } \\
19.4 \pm 1.2 \text { j.r }\end{array}$ & $\begin{array}{c}10033.3 \pm 775.1 \text { a.j } \\
8833.3 \pm 568.6 \text { f.q } \\
8033.3 \pm 1164.4 \text { l.t }\end{array}$ & $\begin{array}{c}3176.7 \pm 648.5 \text { e.m } \\
3085 \pm 118.2 \text { e.n } \\
2736.7 \pm 520.7 \text { j.p } \\
\end{array}$ \\
\hline & & V5 & $\begin{array}{c}\text { Control } \\
50 \% \\
100 \% \\
\end{array}$ & $\begin{array}{c}20.27 \pm 0.23 \text { a.f } \\
20.47 \pm 0.55 \text { a.d } \\
20.73 \pm 0.25 \mathrm{ab}\end{array}$ & $\begin{array}{c}10316.7 \pm 938.5 \text { a.h } \\
9133.3 \pm 1985.8 \text { c.o } \\
10200 \pm 888.8 \text { a.i } \\
\end{array}$ & $\begin{array}{l}3380 \pm 138.1 \text { b.i } \\
3245 \pm 406.1 \text { e. } 1 \\
2825 \pm 135.9 \text { j.o } \\
\end{array}$ \\
\hline & & V7 & $\begin{array}{c}\text { Control } \\
50 \% \\
100 \% \\
\end{array}$ & $\begin{array}{l}20.63 \pm 0.49 \mathrm{abc} \\
20.23 \pm 0.21 \mathrm{a} . \mathrm{g} \\
20.07 \pm 0.59 \mathrm{~b} . \mathrm{k} \\
\end{array}$ & $\begin{array}{c}10733.3 \pm 1106 \text { a.d } \\
9350 \pm 390.5 \text { c.n } \\
7083.3 \pm 775.1 \text { q.w } \\
\end{array}$ & $\begin{array}{c}3286.7 \pm 110.9 \text { c.k } \\
3060 \pm 236.4 \text { f.o } \\
2501.7 \pm 115.4 \text { n.r } \\
\end{array}$ \\
\hline & & R1 & $\begin{array}{c}\text { Control } \\
50 \% \\
100 \% \\
\end{array}$ & $\begin{array}{c}20.4 \pm 0.6 \text { a.e } \\
20.4 \pm 0.1 \text { a.e } \\
19.63 \pm 0.4 \text { f.p }\end{array}$ & $\begin{array}{l}9316.7 \pm 1101.5 \mathrm{c} . \mathrm{n} \\
10400 \pm 2201.7 \mathrm{a} . \mathrm{g} \\
5666.7 \pm 378.6 \mathrm{vwx} \\
\end{array}$ & $\begin{array}{c}3325 \pm 464.9 \text { b.j } \\
3508.3 \pm 431.4 \text { a.g } \\
\mathbf{1 5 8 5} \pm \mathbf{1 9 9 . 8} \mathbf{~ u} \\
\end{array}$ \\
\hline & \multirow{4}{*}{ 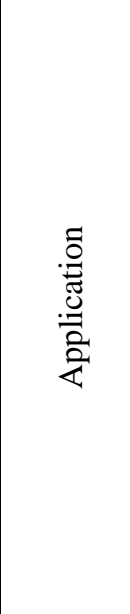 } & V1 & $\begin{array}{c}\text { Control } \\
50 \% \\
100 \% \\
\end{array}$ & $\begin{array}{l}20.47 \pm 0.4 \text { a.d } \\
20.13 \pm 0.81 \text { a.i } \\
19.67 \pm 0.71 \text { f.p }\end{array}$ & $\begin{array}{c}10900 \pm 624.5 \mathrm{abc} \\
\mathbf{1 1 7 5 0} \pm \mathbf{3 0 0} \text { a } \\
8716.7 \pm 361.7 \text { g.p }\end{array}$ & $\begin{array}{c}3875 \pm 360.9 \mathrm{abc} \\
3895 \pm 82.3 \mathrm{ab} \\
3481.7 \pm 311.3 \mathrm{a.g} \\
\end{array}$ \\
\hline & & V3 & $\begin{array}{c}\text { Control } \\
50 \% \\
100 \% \\
\end{array}$ & $\begin{array}{c}19.37 \pm 0.91 \text { k.r } \\
19.53 \pm 0.91 \text { g.q } \\
19.47 \pm 0.21 \text { i.r }\end{array}$ & $\begin{array}{c}10533.3 \pm 1183.6 \text { a.f } \\
10300 \pm 1175.8 \text { a.h } \\
9566.7 \pm 513.2 \text { b.m } \\
\end{array}$ & $\begin{array}{l}3468.3 \pm 363 \text { a.g } \\
3440 \pm 122.9 \text { b.h } \\
3330 \pm 312.8 \text { b.j } \\
\end{array}$ \\
\hline & & V5 & $\begin{array}{c}\text { Control } \\
50 \% \\
100 \% \\
\end{array}$ & $\begin{array}{c}20.47 \pm 0.7 \text { a.d } \\
19.63 \pm 0.67 \text { f.p } \\
19.63 \pm 0.12 \text { f.p } \\
\end{array}$ & $\begin{array}{c}10666.7 \pm 1056.3 \text { a.e } \\
\mathbf{1 1 7 3 3 . 3} \pm \mathbf{2 6 7 0 . 4} \mathbf{~ a} \\
10366.7 \pm 1661.6 \text { a.h }\end{array}$ & $\begin{array}{c}3658.3 \pm 444.6 \text { a.e } \\
\mathbf{4 0 4 0} \pm \mathbf{9 1 2} \text { a } \\
3495 \pm 373.6 \text { a.g }\end{array}$ \\
\hline & & V7 & $\begin{array}{c}\text { Control } \\
50 \% \\
100 \%\end{array}$ & $\begin{array}{l}19.67 \pm 0.29 \text { f.p } \\
19.9 \pm 0.44 \text { d.m } \\
19.03 \pm 0.74 \text { p.s }\end{array}$ & $\begin{array}{c}11300 \pm 1361.1 \mathrm{ab} \\
9700 \pm 526.8 \mathrm{~b} .1 \\
7800 \pm 492.4 \mathrm{~m} . \mathrm{u}\end{array}$ & $\begin{array}{l}3906.7 \pm 162.6 \mathrm{ab} \\
3353.3 \pm 265.4 \mathrm{~b} . \mathrm{i} \\
2518.3 \pm 516.2 \mathrm{n} . \mathrm{r}\end{array}$ \\
\hline
\end{tabular}




\begin{tabular}{|c|c|c|c|c|c|c|}
\hline & & $\mathrm{R} 1$ & $\begin{array}{c}\text { Control } \\
50 \% \\
100 \%\end{array}$ & $\begin{array}{l}19.47 \pm 0.32 \text { i.r } \\
19.87 \pm 0.15 \text { d.n } \\
19.23 \pm 0.68 \text { m.s }\end{array}$ & $\begin{array}{c}10233.3 \pm 1600.3 \text { a.h } \\
10500 \pm 650 \text { a.g } \\
7450 \pm 1068.9 \text { o.v }\end{array}$ & $\begin{array}{l}3456.7 \pm 308.5 \text { a.h } \\
3456.7 \pm 137.1 \text { a.h } \\
2226.7 \pm 460.6 \text { p.t }\end{array}$ \\
\hline \multirow{10}{*}{ 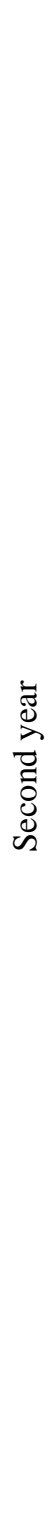 } & \multirow{5}{*}{ 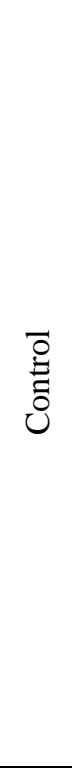 } & V1 & $\begin{array}{c}\text { Control } \\
50 \% \\
100 \% \\
\end{array}$ & $\begin{array}{l}20.4 \pm 0.42 \text { a.e } \\
19.5 \pm 0.42 \text { h.q } \\
19.7 \pm 0.71 \text { e.p }\end{array}$ & $\begin{array}{c}8362.5 \pm 1538 \text { j.s } \\
7700 \pm 70.7 \text { n.u } \\
8350 \pm 883.9 \text { j.s }\end{array}$ & $\begin{array}{c}3030 \pm 169.7 \text { f.o } \\
2500 \pm 70.7 \text { n.r } \\
2925 \pm 388.9 \text { g.o }\end{array}$ \\
\hline & & V3 & $\begin{array}{c}\text { Control } \\
50 \% \\
100 \% \\
\end{array}$ & $\begin{array}{c}20.2 \pm 0.14 \text { a.h } \\
20 \pm 0.28 \text { c.l } \\
19.50 \pm 0.1 \text { h.q }\end{array}$ & $\begin{array}{l}8325 \pm 2333.5 \text { j.s } \\
9025 \pm 424.3 \text { d.o } \\
7937.5 \pm 265.2 \text { 1.t }\end{array}$ & $\begin{array}{l}2915 \pm 445.5 \text { g.o } \\
3442.5 \pm 449 \text { b.h } \\
2585 \pm 219.2 \text { m.q }\end{array}$ \\
\hline & & V5 & $\begin{array}{c}\text { Control } \\
50 \% \\
100 \% \\
\end{array}$ & $\begin{array}{l}20.1 \pm 0.42 \text { a.j } \\
20.10 \pm 0.1 \text { a.j } \\
18.9 \pm 0.14 \text { qrs }\end{array}$ & $\begin{array}{l}7137.5 \pm 654.1 \text { p.w } \\
9587.5 \pm 2740 \text { b.m } \\
5537.5 \pm 583.4 \text { wx }\end{array}$ & $\begin{array}{c}2475 \pm 530.3 \text { o.s } \\
2935 \pm 827.3 \text { g.o } \\
\mathbf{1 4 7 0} \pm \mathbf{1 1 . 1} \mathbf{~ u} \\
\end{array}$ \\
\hline & & V7 & $\begin{array}{c}\text { Control } \\
50 \% \\
100 \% \\
\end{array}$ & $\begin{array}{l}19.9 \pm 0.14 \text { d.m } \\
19.7 \pm 0.14 \text { e.p } \\
19.10 \pm 0.1 \text { o.s } \\
\end{array}$ & $\begin{array}{c}8187.5 \pm 2103.6 \mathrm{k.t} \\
6037.5 \pm 1043 \text { u.x } \\
6412.5 \pm 300.5 \text { t.x }\end{array}$ & $\begin{array}{c}2700 \pm 495 \text { k.p } \\
1882.5 \pm 371.2 \mathrm{stu} \\
1955 \pm 268.7 \mathrm{r.u} \\
\end{array}$ \\
\hline & & R1 & $\begin{array}{c}\text { Control } \\
50 \% \\
100 \% \\
\end{array}$ & $\begin{array}{c}19.4 \pm 0.28 \mathrm{j} . \mathrm{r} \\
19.25 \pm 0.64 \mathrm{~m} . \mathrm{s} \\
18.75 \pm 0.07 \mathrm{rs}\end{array}$ & $\begin{array}{c}8425 \pm 777.8 \text { i.s } \\
7512.5 \pm 441.9 \text { p.u } \\
\mathbf{5 2 2 5} \pm \mathbf{1 3 7 8 . 9} \mathbf{x}\end{array}$ & $\begin{array}{c}3085 \pm 120.2 \text { e.n } \\
2660 \pm 14.1 \text { l.p } \\
\mathbf{1 5 2 5} \pm \mathbf{2 4 7 . 5} \text { u }\end{array}$ \\
\hline & \multirow{5}{*}{ 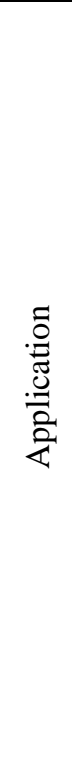 } & V1 & $\begin{array}{c}\text { Control } \\
50 \% \\
100 \% \\
\end{array}$ & $\begin{array}{c}19.88 \pm 0.45 \text { d.m } \\
19.8 \pm 0.32 \text { d.o } \\
20.03 \pm 0.22 \text { b. } 1\end{array}$ & $\begin{array}{c}8018.8 \pm 1049.9 \text { l.t } \\
8750 \pm 1589.4 \text { f.q } \\
9156.3 \pm 1826.2 \text { c.o }\end{array}$ & $\begin{array}{l}3196.3 \pm 512.6 \text { e. } 1 \\
3263.8 \pm 210.8 \text { d.k } \\
3213.8 \pm 613.1 \text { e. } 1\end{array}$ \\
\hline & & V3 & $\begin{array}{c}\text { Control } \\
50 \% \\
100 \% \\
\end{array}$ & $\begin{array}{c}20.33 \pm 0.63 \text { a.f } \\
19.65 \pm 0.4 \text { f.p } \\
19.15 \pm 0.57 \text { n.s }\end{array}$ & $\begin{array}{c}9962.5 \pm 1379.2 \text { a.k } \\
8900 \pm 1479.6 \text { e.p } \\
8362.5 \pm 948.1 \text { j.s }\end{array}$ & $\begin{array}{c}3388.8 \pm 395.8 \text { b.i } \\
3160 \pm 338.7 \text { e.m } \\
2825 \pm 445.5 \text { i.o }\end{array}$ \\
\hline & & V5 & $\begin{array}{c}\text { Control } \\
50 \% \\
100 \% \\
\end{array}$ & $\begin{array}{c}20.08 \pm 0.25 \text { b.k } \\
19.48 \pm 0.97 \text { i.q } \\
\mathbf{1 8 . 5 8} \pm \mathbf{0 . 3 2} \text { s }\end{array}$ & $\begin{array}{c}7962.5 \pm 592.5 \text { 1.t } \\
8406.3 \pm 1128.7 \text { j.s } \\
6668.8 \pm 2115.6 \text { s.x }\end{array}$ & $\begin{array}{l}2866.3 \pm 558.7 \text { h.o } \\
3021.3 \pm 439.1 \text { g.o } \\
1992.5 \pm 540.5 \text { q.u }\end{array}$ \\
\hline & & V7 & $\begin{array}{c}\text { Control } \\
50 \% \\
100 \% \\
\end{array}$ & $\begin{array}{c}19.63 \pm 0.34 \text { f.p } \\
19.7 \pm 0.64 \text { e.p } \\
19.13 \pm 0.29 \text { o.s }\end{array}$ & $\begin{array}{c}8593.8909 .1 \text { h.r } \\
8043.8495 .6 \text { l.t } \\
6831.31015 .8 \text { r.x }\end{array}$ & $\begin{array}{c}3180 \pm 579.6 \text { e. } 1 \\
3042.5 \pm 94.3 \text { f.o } \\
2191.3 \pm 430.2 \text { p.t }\end{array}$ \\
\hline & & $\mathrm{R} 1$ & $\begin{array}{l}\text { Control } \\
50 \% \\
100 \%\end{array}$ & $\begin{array}{c}20.23 \pm 0.56 \text { a.g } \\
19.33 \pm 0.26 \text { l.r } \\
19.1 \pm 0.42 \text { o.s }\end{array}$ & $\begin{array}{c}\text { 10737.51667.6 a.d } \\
8581.31498 .1 \text { h.r } \\
5487.51080 .4 \text { wx }\end{array}$ & $\begin{array}{c}3845 \pm 485.6 \text { a.d } \\
2986.3 \pm 358.4 \text { g.o } \\
1687.5 \pm 218.5 \mathrm{tu}\end{array}$ \\
\hline
\end{tabular}

Columns with different letters are significantly different at $\mathrm{P}=0.05$, using LSD test. The bold number showed the highest and lowest means of trait

\section{Grain yield}

The effects of $\mathrm{Y}, \mathrm{AA}, \mathrm{DT}, \mathrm{DI}, \mathrm{Y} \times \mathrm{DT}, \mathrm{DT} \times \mathrm{DI}, \mathrm{Y} \times \mathrm{DT} \times \mathrm{DI}$, and $\mathrm{Y} \times \mathrm{AA} \times \mathrm{DT} \times \mathrm{DI}$ was significant on grain yield (Table 4). In the first $\mathrm{Y}$ (3233.8 $\mathrm{kg}^{-h^{-1}}$ ), AA application (3145.1 kg.ha-1), defoliation in V1 growth stage (3290.4 kg.ha-1), and DI 0 and 50\% (3299.9 and $3213.8 \mathrm{~kg} \cdot \mathrm{ha}^{-1}$, respectively). In the quadruple interaction, the highest grain yield was observed in the application of AA under defoliation in V5 with 50\% intensity during the first year $\left(4040 \mathrm{~kg} \cdot \mathrm{ha}^{-1}\right)$. The lowest value of this trait was achieved in non- 
application of AA under $100 \%$ defoliation in R1 growth stage during both years of experiments (1585 and $1525 \mathrm{~kg} \cdot \mathrm{ha}^{-1}$, respectively) (Table 5).

\section{Harvest index}

The effects of $\mathrm{Y}, \mathrm{AA}, \mathrm{DT}$. DI, $\mathrm{Y} \times \mathrm{DI}, \mathrm{DT} \times \mathrm{DI}$ were significant on harvest index (Table 4). The highest of harvest index was observed in the second Y (34.56\%), AA application $(34.73 \%)$, defoliation in V1 growth stage (35.4\%), 0 and $50 \%$ defoliation intensity (34.85 and $34.80 \%$, respectively) (Table 4). Interaction effect of DT $\times \mathrm{DI}$ showed the highest mean of harvest index in defoliation in V1 and R1 growth stages under control intensity treatments (36.2 and $35.7 \%$, respectively). The lowest value of the trait was observed in $100 \%$ defoliation during the R1 growth stage (29.7\%) (Fig. 8).

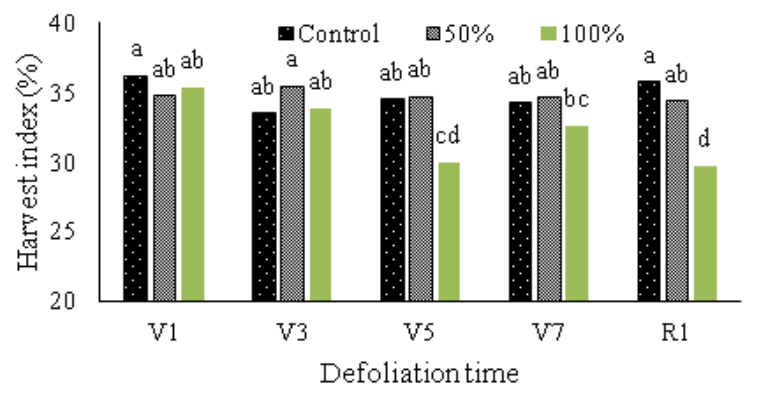

Figure 8. Effect of intensity (0, 50, and 100\%) and time (V1, V3, V5, V7, and R1) of defoliation on harvest index of soybean (columns with different letters are significantly different at

$$
P=0.05 \text {, using LSD test) }
$$

\section{Grain oil content}

Analysis of variance results showed that the effects of $Y$ and $Y \times D T \times D I$ were significant on oil percentage. Also, the effects of $\mathrm{Y}, \mathrm{AA}, \mathrm{DT}, \mathrm{DI}, \mathrm{Y} \times \mathrm{AA}, \mathrm{DT} \times \mathrm{DI}$, $\mathrm{Y} \times \mathrm{AA} \times \mathrm{DT}, \mathrm{Y} \times \mathrm{DT} \times \mathrm{DI}$, and $\mathrm{Y} \times \mathrm{AA} \times \mathrm{DT} \times \mathrm{DI}$ was significant on oil yield $($ Table 6$)$. The highest grain oil yield was achieved in first $\mathrm{Y}\left(726.4 \mathrm{~kg}^{-h a^{-1}}\right)$, AA application (689.7 kg.ha $\left.{ }^{-1}\right)$, defoliation in the V3 growth stage (699.7 kg.ha $\left.{ }^{-1}\right)$, and zero percentage of intensity defoliation (700.5 kg.ha-1) (Table 6). In the quadruple interaction, the maximum grain oil yield was observed in $100 \%$ defoliation in V7 growth stage under AA application during the first-year experiment $\left(978.5 \mathrm{~kg} \cdot \mathrm{ha}^{-1}\right)$. Also, the lowest value of the trait was observed in $100 \%$ defoliation in the V5 growth stage under nonapplication of AA during the second year (225.6 kg.ha-1) (Table 7).

Table 6. Analysis of variance indicating the effects of intensity and time of defoliation on oil yield and percentage and protein yield and percentage under application of amino acid

\begin{tabular}{c|c|c|c|c}
\hline Treatments & Oil percentage & $\begin{array}{c}\text { Oil yield } \\
\left(\text { kg.ha }^{-1}\right)\end{array}$ & $\begin{array}{c}\text { Protein } \\
\text { percentage }\end{array}$ & $\begin{array}{c}\text { Protein yield } \\
\left(\text { kg.ha-1 }^{-1}\right)\end{array}$ \\
\hline Year $(\mathbf{Y})$ & & & & \\
First & $22.3 \mathrm{a}$ & $726.4 \mathrm{a}$ & $33.66 \mathrm{a}$ & $1087.55 \mathrm{a}$ \\
Second & $19.9 \mathrm{~b}$ & $564.8 \mathrm{~b}$ & $31.23 \mathrm{a}$ & $874.10 \mathrm{~b}$ \\
LSD $(P=0.05)$ & 2.01 & 71.2 & 2.08 & 109.5 \\
\hline
\end{tabular}




\begin{tabular}{|c|c|c|c|c|}
\hline \multicolumn{5}{|l|}{ Amino acid (AA) } \\
\hline Control & $20.68 \mathrm{a}$ & $583.8 \mathrm{~b}$ & $32.94 \mathrm{a}$ & $921.9 \mathrm{~b}$ \\
\hline Application & $21.48 \mathrm{a}$ & $689.7 \mathrm{a}$ & $32.09 \mathrm{a}$ & $1022.9 \mathrm{a}$ \\
\hline $\operatorname{LSD}(P=0.05)$ & 1.53 & 81.1 & 1.49 & 49.9 \\
\hline \multicolumn{5}{|l|}{ Defoliation time (DT) } \\
\hline $\mathrm{V} 1$ & $20.26 \mathrm{a}$ & $649.1 \mathrm{~b}$ & $31.55 \mathrm{a}$ & $996.1 \mathrm{~b}$ \\
\hline V3 & $21.81 \mathrm{a}$ & $699.7 \mathrm{a}$ & $32.87 \mathrm{a}$ & $1053.8 \mathrm{a}$ \\
\hline V5 & $20.96 \mathrm{a}$ & $606.5 \mathrm{c}$ & $31.78 \mathrm{a}$ & $904.0 \mathrm{c}$ \\
\hline V7 & $21.62 \mathrm{a}$ & $658.2 \mathrm{~b}$ & $33.30 \mathrm{a}$ & $1004.3 \mathrm{~b}$ \\
\hline $\mathrm{R} 1$ & $21.09 \mathrm{a}$ & $614.6 \mathrm{c}$ & $32.73 \mathrm{a}$ & $945.8 \mathrm{c}$ \\
\hline $\operatorname{LSD}(P=0.05)$ & 1.11 & 12.49 & 2.07 & 49.8 \\
\hline \multicolumn{5}{|c|}{ Defoliation intensity (DI) (\%) } \\
\hline 0 & $21.38 \mathrm{a}$ & $700.5 \mathrm{a}$ & $32.2 \mathrm{a}$ & $1052.0 \mathrm{a}$ \\
\hline 50 & $21.26 \mathrm{a}$ & $650.1 \mathrm{~b}$ & $32.4 \mathrm{a}$ & $984.5 \mathrm{a}$ \\
\hline 100 & $20.81 \mathrm{a}$ & $586.3 \mathrm{c}$ & $32.6 \mathrm{a}$ & $905.8 \mathrm{~b}$ \\
\hline $\operatorname{LSD}(P=0.05)$ & 1.13 & 52.3 & 0.86 & 77.2 \\
\hline \multicolumn{5}{|l|}{ Interaction effect } \\
\hline $\mathrm{Y} \times \mathrm{AA}$ & NS & $*$ & NS & NS \\
\hline $\mathrm{Y} \times \mathrm{DT}$ & NS & NS & NS & NS \\
\hline $\mathrm{Y} \times \mathrm{DI}$ & NS & NS & NS & NS \\
\hline $\mathrm{AA} \times \mathrm{DT}$ & NS & NS & NS & NS \\
\hline $\mathrm{AA} \times \mathrm{DI}$ & NS & NS & NS & NS \\
\hline $\mathrm{DT} \times \mathrm{DI}$ & NS & $*$ & NS & $*$ \\
\hline $\mathrm{Y} \times \mathrm{AA} \times \mathrm{DT}$ & NS & $*$ & NS & NS \\
\hline $\mathrm{Y} \times \mathrm{AA} \times \mathrm{DI}$ & NS & NS & NS & $*$ \\
\hline $\mathrm{Y} \times \mathrm{DT} \times \mathrm{DI}$ & $*$ & $* *$ & NS & $*$ \\
\hline $\mathrm{AA} \times \mathrm{DT} \times \mathrm{DI}$ & NS & $* *$ & NS & $* *$ \\
\hline $\mathrm{Y} \times \mathrm{AA} \times \mathrm{DT} \times \mathrm{DI}$ & NS & $*$ & NS & * \\
\hline
\end{tabular}

LSD: least significant difference; NS: non-significant. Columns with different letters are significantly different at $\mathrm{P}=0.05$, using LSD test

\section{Grain protein content}

Results indicated that the effects of $\mathrm{Y}, \mathrm{AA}, \mathrm{DT}, \mathrm{DI}, \mathrm{DT} \times \mathrm{DI}, \mathrm{Y} \times \mathrm{AA} \times \mathrm{DI}, \mathrm{Y} \times \mathrm{DT} \times$ $\mathrm{DI}, \mathrm{AA} \times \mathrm{DT} \times \mathrm{DI}$, and $\mathrm{Y} \times \mathrm{AA} \times \mathrm{DT} \times \mathrm{DI}$ were significant on grain protein yield (Table 6). In the quadruple interaction, the highest grain protein yield was observed in $100 \%$ defoliation in V7 growth stage under AA application during the first-year experiment (1499 kg.ha-1). Also, the lowest value of the trait was observed in $100 \%$ defoliation in the V5 growth stage under non-application of AA during the second year (471.9 kg.ha-1) (Table 7). 
Table 7. Interaction effects of year, amino acid, defoliation time, and defoliation intensity on grain oil and protein yield of soybean

\begin{tabular}{|c|c|c|c|c|c|}
\hline Year & Amino acid & Defoliation time & $\begin{array}{l}\text { Defoliation } \\
\text { intensity }\end{array}$ & $\begin{array}{c}\text { Grain oil yield } \\
\left(\mathrm{kg}^{-h^{-1}}\right)\end{array}$ & \begin{tabular}{|c|} 
Grain protein yield \\
$\left(\right.$ kg.ha $\left.^{-1}\right)$
\end{tabular} \\
\hline \multirow{30}{*}{ 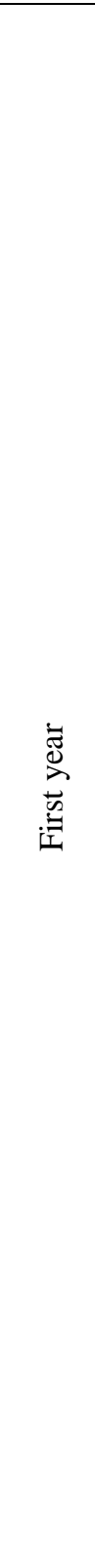 } & \multirow{15}{*}{$\begin{array}{l}\overline{0} \\
\text { 苛 }\end{array}$} & \multirow{3}{*}{$\mathrm{V} 1$} & Control & $606.7 \pm 173.7$ g.n & $1068.6 \pm 110.6$ b.n \\
\hline & & & $50 \%$ & $607.9 \pm 131.1$ g.n & $954 \pm 202.5$ f.q \\
\hline & & & $100 \%$ & $515.6 \pm 168.4$ k.q & $896.5 \pm 310.1$ h.q \\
\hline & & \multirow{3}{*}{ V3 } & Control & $685.7 \pm 111.1 \mathrm{c} . \mathrm{k}$ & $1004 \pm 150.5$ d.p \\
\hline & & & $50 \%$ & $719.7 \pm 92.3$ b.i & $1121 \pm 206.8$ b.k \\
\hline & & & $100 \%$ & $768.5 \pm 94$ b.h & $1077.9 \pm 110.7$ b.m \\
\hline & & \multirow{3}{*}{ V5 } & Control & $822.7 \pm 87.8$ a.e & $1188.2 \pm 223$ b.f \\
\hline & & & $50 \%$ & $617.9 \pm 31.9$ a.e & $940.9 \pm 44$ f.q \\
\hline & & & $100 \%$ & $495.9 \pm 162.8$ k.q & $869.5 \pm 338.8$ k.q \\
\hline & & \multirow{3}{*}{ V7 } & Control & $802.4 \pm 67.2$ a.f & $1239.7 \pm 160.9$ b.e \\
\hline & & & $50 \%$ & $670.4 \pm 178.3 \mathrm{c} .1$ & $1024.2 \pm 240.9$ d.o \\
\hline & & & $100 \%$ & $731.1 \pm 333.6$ b.i & $1073.6 \pm 436.3$ b.n \\
\hline & & \multirow{3}{*}{$\mathrm{R} 1$} & Control & 595.8174 h.n & $880.4 \pm 254.6$ i.q \\
\hline & & & $50 \%$ & $570.7 \pm 107$ i.n & $936.8 \pm 223.8$ f.q \\
\hline & & & $100 \%$ & $667.1 \pm 225.6$ c.m & $1057.5 \pm 345.6$ b.o \\
\hline & \multirow{15}{*}{ 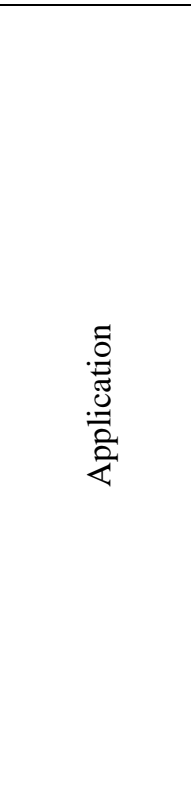 } & & Control & $825.2 \pm 121.4$ a.d & $1138.7 \pm 164.2$ b.h \\
\hline & & V1 & $50 \%$ & $849.8 \pm 95.5 \mathrm{abc}$ & $1116.5 \pm 186.9$ b.k \\
\hline & & & $100 \%$ & $768 \pm 72.7$ b.h & $1120.4 \pm 150.4$ b.k \\
\hline & & & Control & $882.2 \pm 91.8 \mathrm{ab}$ & $1297.2 \pm 186.4 \mathrm{ab}$ \\
\hline & & V3 & $50 \%$ & $725.4 \pm 110.8$ b.i & $1147.6 \mathrm{v} 124.1 \mathrm{~b} . \mathrm{h}$ \\
\hline & & & $100 \%$ & $776.8 \pm 95.4$ b.h & $1125.9 \pm 234.3$ b.j \\
\hline & & & Control & $804.9 \pm 118.6$ a.f & $1109.4 \pm 97.2$ b.k \\
\hline & & V5 & $50 \%$ & $828 \pm 73.5$ a.d & $1133.1 \pm 69.6$ b.i \\
\hline & & & $100 \%$ & $654.8 \pm 116.1$ d.m & $987 \pm 69.1$ e.p \\
\hline & & & Control & $769.3 \pm 145.9$ b.h & $1140.2 \pm 265.9$ b.h \\
\hline & & V7 & $50 \%$ & $732.7 \pm 130$ b.i & $1075.4 \pm 173.1$ b.m \\
\hline & & & $100 \%$ & $978.5 \pm 170.5$ a & $1499 \pm 275.2 \mathrm{a}$ \\
\hline & & & Control & $791.3 \pm 61.7$ a.g & $1162.1 \pm 120.2$ b.g \\
\hline & & $\mathrm{R} 1$ & $50 \%$ & $636.3 \pm 39.8$ d.n & $951.6 \pm 59.5$ f.q \\
\hline & & & $100 \%$ & $892.7 \pm 208.4 \mathrm{ab}$ & $1289.6 \pm 301 \mathrm{abc}$ \\
\hline \multirow{9}{*}{ 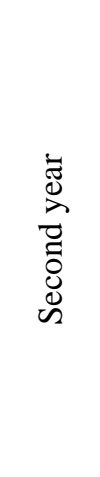 } & \multirow{9}{*}{ 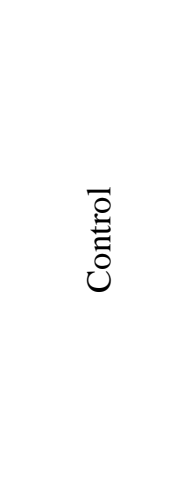 } & & Control & $522.9 \pm 17.8$ j.p & $962.1 \pm 163$ f.q \\
\hline & & V1 & $50 \%$ & $474 \pm 143.9 \mathrm{~m} . \mathrm{r}$ & $727 \pm 101.4$ q.u \\
\hline & & & $100 \%$ & $458.2 \pm 175.7$ n.r & $819.7 \pm 76.2$ n.r \\
\hline & & & Control & $517.3 \pm 77$ k.q & $812.7 \pm 97.7$ o.r \\
\hline & & V3 & $50 \%$ & $683.2 \pm 139.8$ c.k & $1075.3 \pm 133$ b.m \\
\hline & & & $100 \%$ & $560.7 \pm 79.9$ i.n & $828.1 \pm 30.2 \mathrm{~m} . \mathrm{r}$ \\
\hline & & & Control & $540 \pm 74.7$ i.o & $769 \pm 214.4$ p.t \\
\hline & & V5 & $50 \%$ & $543.5 \pm 207$ i.o & $876.4 \pm 318.8$ j.q \\
\hline & & & $100 \%$ & $225.6 \pm 57.2 \mathrm{~s}$ & $471.9 \pm 14.6 \mathrm{v}$ \\
\hline
\end{tabular}




\begin{tabular}{|c|c|c|c|c|}
\hline & V7 & $\begin{array}{c}\text { Control } \\
50 \% \\
100 \% \\
\end{array}$ & $\begin{array}{c}565.9 \pm 135.7 \text { i.n } \\
355.7 \pm 97.6 \text { o.s } \\
325.2 \pm 110.4 \text { qrs }\end{array}$ & $\begin{array}{c}915.7 \pm 85.3 \text { g.q } \\
604 \pm 134.8 \text { r.v } \\
554.2 \pm 117.2 \text { s.v }\end{array}$ \\
\hline & $\mathrm{R} 1$ & $\begin{array}{c}\text { Control } \\
50 \% \\
100 \% \\
\end{array}$ & $\begin{array}{l}521.4 \pm 136.6 \text { j.p } \\
489.8 \pm 130.5 \text { l.q } \\
294.7 \pm 107.4 \text { rs }\end{array}$ & $\begin{array}{c}804.1 \pm 25.3 \text { o.s } \\
848.7 \pm 68.5 \text { l.r } \\
503.3 \pm 211.5 \mathrm{uv}\end{array}$ \\
\hline \multirow{5}{*}{ 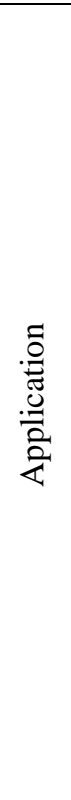 } & V1 & $\begin{array}{c}\text { Control } \\
50 \% \\
100 \%\end{array}$ & $\begin{array}{c}630.9 \pm 211.1 \text { e.n } \\
713 \pm 216.4 \text { b.j } \\
640.7 \pm 208.4 \text { d.n }\end{array}$ & $\begin{array}{c}960.1 \pm 138.3 \text { f.q } \\
1011.9 \pm 113.3 \text { d.p } \\
1017.5 \pm 196.7 \text { d.p }\end{array}$ \\
\hline & V3 & $\begin{array}{c}\text { Control } \\
50 \% \\
100 \%\end{array}$ & $\begin{array}{l}731.1 \pm 137.4 \text { b.i } \\
629.9 \pm 114.9 \text { e.n } \\
637.5 \pm 205.5 \text { d.n }\end{array}$ & $\begin{array}{c}1087.4 \pm 181 \text { b.l } \\
1041.8 \pm 95.9 \text { c.o } \\
917 \pm 196.4 \text { g.q }\end{array}$ \\
\hline & V5 & $\begin{array}{c}\text { Control } \\
50 \% \\
100 \% \\
\end{array}$ & $\begin{array}{l}624.1 \pm 122.5 \text { f.n } \\
674.4 \pm 173.2 \text { c.l } \\
337.9 \pm 126.7 \text { p.s }\end{array}$ & $\begin{array}{l}894.2 \pm 91.8 \text { h.q } \\
958.7 \pm 162.3 \text { f.q } \\
553.6 \pm 166.3 \text { s.v }\end{array}$ \\
\hline & V7 & $\begin{array}{c}\text { Control } \\
50 \% \\
100 \% \\
\end{array}$ & $\begin{array}{c}673.7 \pm 162.1 \text { c.l } \\
657.365 .2 \text { c.m } \\
456.594 \text { n.r }\end{array}$ & $\begin{array}{c}1032.5 \pm 213.4 \text { d.o } \\
963.9 \pm 60.2 \text { f.q } \\
716.4 \pm 133.4 \text { q.v }\end{array}$ \\
\hline & $\mathrm{R} 1$ & $\begin{array}{c}\text { Control } \\
50 \% \\
100 \%\end{array}$ & $\begin{array}{c}824.9157 .2 \text { a.d } \\
584.7193 .2 \text { h.n } \\
353.772 .7 \text { o.s }\end{array}$ & $\begin{array}{c}1253.3 \pm 184.4 \text { a.d } \\
925.4 \pm 170.3 \text { g.q } \\
547.4 \pm 75.3 \text { tuv }\end{array}$ \\
\hline
\end{tabular}

Columns with different letters are significantly different at $\mathrm{P}=0.05$, using LSD test. The bold number showed the highest and lowest means of trait

\section{Discussion}

\section{Effect of defoliation time and intensity}

Defoliation caused by insects is common biotic stress in soybean [Glycine max (L.) Merr.] production (Xiangjun et al., 2009). The effects of defoliation on yield and yield components of soybean have been well studied, but time and intensity of defoliation have not been demonstrated. Defoliation affected soybean yield through a combination of reduced light interception resulting in decreased canopy photosynthesis, loss of leaf storage material, and/or shortening of the effective grain-filling period (Xiangjun et al., 2006). In the experiments of Xianjun et al. (2006, 2009), the effect of defoliation of soybean under salinity stress or application of nitrogen fertilizer were investigated. The effect of defoliation on growth and yield characteristics in plants varied with time and intensity of defoliation (Xiangjun et al., 2009). In the present study, effects of time and intensity of defoliation were significant on morphological traits, and yield and yield components such as plant height, first pod height, numbers of lateral and main branch, number of total pod, number of grain, 100-grain weight, biological yield, grain yield, and harvest index (Tables 3 and 4). Results illustrated by increases defoliation intensity was observed decreasing on yield and yield components. Also, defoliation in the reproductive growth stage (R1) had significantly reduced on studied traits. Several studies have shown strong relationships between the leaf-area index, defoliation intensity, light interception rate, and yield (Hammond et al., 2000; Xiangjun et al., 
2006). Defoliation decreased soybean dry-matter production by reducing the effective leaf area for light interception and carbon fixation (Klubertanz et al., 1996). On the other, it may enhance growth through two mechanisms: compensatory regrowth and delayed leaf senescence, including delayed leaf abscission and increased leaf photosynthetic rates (Xiangjun et al., 2006). These mechanisms may aid soybean in tolerating defoliation during vegetative and early reproductive stages; however, the ability of these mechanisms to function in the presence of additional stresses is not known. In this regard, Ugese et al. (2011) reported that the defoliation time and intensity significantly affected on seedlings of Vitellaria paradoxa, which confirms the results of this study. Non-defoliation and mild defoliation intensity (50\%) would not have a significant effect on yield reduction, but the severe defoliation intensity $(100 \%)$ was caused decreasing in the studied traits. In this regards, the yield and growth reduction under severe stress conditions (100\% defoliation) can be attributed to reduced photosynthetic parameters (net photosynthesis, transpiration rate, stomatal conductance, and chlorophyll content) (Zobiole et al., 2009, 2010). Defoliations in the early stages of growth apparently does not affect yield and yield components positively or negatively, an observation that is consistent with other types of crops, such as corn and garlic (Olfati et al., 2010). Xiangjun et al. (2009) reported that although defoliation temporarily reduced soybean dry weight and $\mathrm{N}$ accumulation during 15 days after defoliation.

Our finding that increasing levels of defoliation leads to progressive declines in grain oil and protein yield, but not significantly affected protein percentage (Table 6). This result agreed with the report by Turnipseed (1972) and Xiangjun et al. (2009) and that defoliation caused no decrease in seed protein content. Nitrogen (one of the important elements in protein synthesis) absorption following defoliation may be promoted. Although root growth usually is reduced following defoliation, the increase in nutrient uptake rate per unit root mass was found in a sedge (Kyllinga nervosa Steud.) plants (McNaughton and Chapin, 1985).

\section{Effect of amino acid application}

Amino acids are molecules with the following structure: a central carbon atom (C), usually asymmetric, attached to a carboxylic acid group $(\mathrm{COOH})$, an amino group amidogen $\left(\mathrm{NH}_{2}\right)$ and hydrogen $(\mathrm{H})$ atom (Moreira and Moraes, 2017). The AAs have several functions, and the most important are: i) protein synthesis, ii) intermediate compounds in the synthesis of endogenous plant hormones, iii) chelating effect on nutrients and other compounds, iv) greater resistance to drought stress and high temperatures, and v) greater disease resistance (Castro, 2009; Moreira and Moraes, 2017). In the current study, results indicated that the effect of the amino acid application was significant on yield and yield components as well as oil and protein yield (Tables 4 and 6). Application of amino acid significantly increased plant height, biological yield, grain yield, harvest index, and oil and protein yield. It is well documented that plants are capable of utilizing amino acids as nitrogen $(\mathrm{N})$ and carbon $(\mathrm{C})$ sources (Thornton and Robinson, 2005). Amino acid foliar fertilization has generally been sprayed onto plants to increase the crop yield (Souza et al., 2018). Amino acid foliar applications are biostimulants in plants because they enhance the nutrient uptake efficiency because of increases in the leaf cuticle permeability (Moreira and Moraes, 2017), better plant growth, and higher plant biomass and grain yield, and they reduce abiotic stresses (Azimi et al., 2013; Gazola et al., 2014; Mendes et al., 2016). Moreira and Moraes 
(2017) found increases in common bean (Phaseolus vulgaris L.) yield after applying amino acid. Despite demonstrating these positive results regarding the efficient use of amino acids, some studies indicated that plant responses may vary under the influence of many factors such as the molecular weight of amino acids, anatomical features, growth stage, climate conditions and time of application (Fernández et al., 2013).

In all studied traits, the means of the traits from each growing season was different (Tables 3, 4, and 6). We found $3233.8 \mathrm{~kg}^{-h^{-1}}$ (first year) and $2795.6 \mathrm{~kg} \cdot \mathrm{ha}^{-1}$ (second year) which means a reduction of $13 \%$ in the grain yield (Table 4). Similar to wheat and soybean, this reduction was caused by hydric stress at the beginning of the soybean development because this stage is the crucial moment for successful crop development, and the effects reflect on crop yield (Souza et al., 2018).

\section{Conclusion}

Results indicated that the defoliation time and intensity significantly decreased morphological traits and yield components as well as oil and protein contents. Server defoliation intensity (100\%) during the R1 growth stage (reproductive stage) showed an intensive reduction effect. While defoliation in early growth stages had a negligible effect on the traits studied. On the other hand, the use of low molecular weight amino acid is aimed to increase nutrient uptake by leaves, with the consequent increase in productivity. The findings of this study showed that, on the average of two years of assessment, the foliar application of amino acid increased morphological traits, yield, and yield components. According to the present study, soybean is regarded generally as a defoliation-tolerant crop. Also, foliar application of amino acid could be used as an effective amendment for preventing defoliation injury in yield and yield components of soybean.

\section{REFERENCES}

[1] Ahmadi, A., Joudi, M., Janmahammadi, M. (2009): Late defoliation and wheat yield: Little evidence of post-anthesis source limitation. - Fields Crop Res 113: 90-93.

[2] Alhamad, M. N., Alrababah, M. A. (2008): Defoliation and competition effects in a productivity gradient for a semiarid Mediterranean annual grassland community. - Basic Appl. Ecol. 9: 224-232.

[3] An, L. V., Frankow-Linberg, B. E., Lindberg, J. E. (2003): Effect of harvesting interval and defoliation on yield and chemical composition of leaves, stems, and tubers of sweet potato. - Field Crop Res. 82: 49-58.

[4] Arslan, H., Karakus, M., Hatipoglu, H., Arslan, D., Bayraktor, O. V. (2018): Assessment of performances of yield and factors affecting the yield in some soybean varieties/lines grown under semi-arid climate conditions. - Applied Ecology and Environmental Research 16(4): 4289-4298.

[5] Azimi, M. S., Daneshian, J., Sayfzadeh, S., Zare, S. (2013): Evaluation of amino acid and salicylic acid application on yield and growth of wheat under water deficit. International Journal of Agriculture Crop Sciences 5: 816-19.

[6] Castro, P. R. C. (2009): Principles of Foliar Fertilization [Princípios da adubação foliar]. - FINEP, Jaboticabal, Brazil.

[7] Divsalar, M., Tahmasebi Sarvestani, Z., Modares Sanavi, S. A. M., Hamidi, A. (2015): Effect of drought stress on protein, oil, and soybean fatty acid composition. - Plant Ecophysiology 27(8): 55-44. 
[8] Fernandez, V., Sotiropoulos, T., Brown, P. (2013): Foliar Fertilization; Scientific Principles and Field Practices. - IFA, Paris.

[9] Gazola, D., Zucareli, C., Silva, R. R., Fonseca, I. D. B. (2014): Foliar application of aminoacids and nitrogen as topdressing on second corn crop. - Revista Brasileira De Engenharia Agrícola E Ambiental 18: 700-07. DOI: 10.1590/S141543662014000700005.

[10] Hammond, R. B., Higley, L. G., Pedigo, L. P., Bledsoe, L., Spomer, S. M., Degooyer, T. A. (2000): Simulated insect defoliation on soybean: Influence of row width. - Journal of Economic Entomology 93: 1429-1436.

[11] Hymowitz, T., Shurtleff, W. R. (2005): Debunking soybean myths and legends in historical and popular literature. - Crop Science 45(2): 473-476.

[12] Khaliliaqdam, N., Soltani, A., Mir-Mohamoodi, T., Jadidi, T. (2012): Effect of leaf defoliation on some agronomical traits of corn. - World Applied Sciences Journal 20(4): 545-548.

[13] Klubertanz, T. H., Pedigo, L. P., Carlson, R. C. (1996): Soybean physiology, regrowth, and senescence in response to defoliation. - Agronomy Journal 88: 577-582.

[14] Kowalczyk, K., Zielony, T. (2008): Effect of aminoplant and asahi on yield and quality of lettuce grown on rockwool. - Conf. of Biostimulators in Modern Agriculture, 7-8 February 2008, Warsaw, Poland.

[15] McNaughton, S. J., Chapin, F. S. (1985): Effects of phosphorus nutrition and defoliation on C4 graminoids from Serengeti Plains. - Ecology 66: 1617-1629.

[16] McWilliam, D. A., Berglund, D. R., Endres, G. J. (1999): Soybean Growth and Management Quick Guide, A-1174. - North Dakota State University, Frago, ND.

[17] Mendes, R. T., Resende, R. C., Pereira, M. A. M., Bento, R. U., Silva, R. C. D., Cruz, S. J. S., Pelá, A. (2016): Foliar application of urea and bell pepper amino acids. - African Journal of Agricultural Research 11: 1674-78. DOI: 10.5897/AJAR2015.10496.

[18] Moreira, A., Moraes, L. A. C. (2017): Yield, nutritional status and soil fertility cultivated with common bean in response to amino-acids foliar application. - Journal of Plant Nutrition 40: 344-351. DOI: 10.1080/01904167.2016.1240194.

[19] Nardino, M., Souza, V. Q., Bahry, C. A., Carbalho, I. R., Caron, B. O., Zimmer, P. D., Busanello, C. (2016): Defoliation at different vegetative stages and assessment of morphological and yield components of soybean. - Current Agricultural Science and Technology 22: 24-32.

[20] Olfati, J. A., Peyvast, Gh., Sanavi, M., Salehi, M., Mahdipour, M. (2010): Effect of defoliation on the yield and quality of garlic. - Journal of Herbs, Spices \& Medicinal Plants 16(2): 91-97. DOI: 10.1080/10496475.2010.481917.

[21] Pickle, C. S., Caviness, C. E. (2006): Yield reduction from defoliation and plant cutoff of determinate and semi-determinate soybean. - Agronomy Journal 76(3): 474-476.

[22] Sadak, S. H. M., Abdelhamid, M. T., Schmidhalter, U. (2015): Effect of foliar application of aminoacids on plant yield and physiological parameters in bean plants irrigated with seawater. - Acta Biol. Colomb. 20(1): 141-152.

[23] Saeed, M. R., Kheir, A. M., Al-Sayed, A. A. (2005): Suppressive effect of some amino acids against Meloidogyne incognita on soybeans. - J. Agric. Sci. Mansoura Univ. 30(2): 1097-1103.

[24] Souza, J. A., Fratoni, M. M. J., Moraes, L. A. C., and Moreira, A. (2018): Boron and amino acid foliar application on wheat-soybean intercropping in a non-tillage system. Communications in Soil Science and Plant Analysis. DOI: 10.1080/00103624.2018.1474902.

[25] Stiegler, J. C., Richardson, M. D., Karcher, D. E., Roberts, T. L., Richard, J., Norman, R. J. (2013): Foliar absorption of various inorganic and organic nitrogen sources by creeping bentgrass. - Crop Sci. 53(3): 1148-1152.

[26] Thornton, B., Robinson, D. (2005): Uptake and assimilation of nitrogen from solutions containing multiple N sources. - Plant Cell and Environment 28: 813-821. 
[27] Turinpseed, S. G. (1972): Response of soybean to foliage losses in South Carolina. Journal of Economic Entomology 60: 1054-1056.

[28] Ugese, F. D., Ahen, A., Ishar, S. S. (2011): Single defoliation had little influence on growth and dry matter attributes of Shea (Vitellaria paradoxa C. F. Gaertn.) seedlings. Forests, Trees and Livelihoods 20: 283-294.

[29] Xiangjun, L., Ping, A., Shinobu, I., Egrinya, E., Xiaojing, L. (2009): Nitrogen accumulation in soybean following defoliation. - Journal of Plant Nutrition 32(1): 71-83. DOI: $10.1080 / 01904160802531027$.

[30] Xiangjun, L., Ping, A., Shinobu, I., Egrinya, E., Kenji, T. (2006): Salinity and defoliation effects on soybean growth, - Journal of Plant Nutrition 29(8): 1499-1508. DOI: 10.1080/01904160600837642.

[31] Zobiole, L. H. S., Oliveira, R. S., Huber, D. M., Constantin, J., de Castro, C., de Oliveira, F. A., Oliveira Jr, A. (2009): Glyphosate reduces shoot concentration of mineral nutrients in glyphosate-resistant soybeans. - Plant and Soil 328: 57-69.

[32] Zobiole, L. H. S., Oliveira Jr., R. S., Kremer, R. J., Constantin, J., Yamada, T., de Oliveira, F. A., Oliveira Jr, A. (2010): Effect of glyphosate on symbiotic N2 fixation and nickel concentration in glyphosate-resistant soybeans. - Applied Soil Ecology 44: 176180. 